\title{
Is there still a place for thrombectomy?
}

\author{
Maciej Dąbrowski ${ }^{1}$, Paweł Tyczyński ${ }^{1}$, Maciej Bęćkowski ${ }^{2}$, Adam Witkowski ${ }^{1}$, Andrzej Ciszewski $^{3}$ \\ ${ }^{1}$ Department of Interventional Cardiology and Angiology, Institute of Cardiology, Warsaw, Poland \\ ${ }^{2}$ Department of Coronary Artery Disease, Institute of Cardiology, Warsaw, Poland \\ ${ }^{3}$ Department of Invasive Cardiology, Institute of Cardiology, Warsaw, Poland
}

Adv Interv Cardiol 2016; 12, 1 (43): 68-69 DOI: $10.5114 /$ pwki.2016.56954

Malignancy is known to be a prothrombotic condition, and some antitumor drugs may amplify the hypercoagulable tendency. We present a female patient with cancer, who developed acute coronary syndrome (ACS) due to occlusive intracoronary thrombus without underlying atherosclerosis.

A 59-year-old woman with metastatic breast cancer diagnosed 2 years ago, paraneoplastic syndrome and steroid diabetes was admitted to our institution due to chest discomfort. The patient was previously treated with chemotherapy (trastuzumab and methylprednisolone). The last course was administered 4 months ago. The antithrombotic treatment with enoxaparin had been discontinued 3 weeks before, when an intensive rehabilitation program for cerebellar syndrome was initiated. Based on ST-segment depression in V4-V6 leads in ECG and elevated troponin T up to $2923 \mathrm{ng} / \mathrm{ml}(\mathrm{UNL}<14)$, non-ST segment elevation myocardial infarction (NSTEMI) was diagnosed. Coronary angiography did not show any vessel wall irregularities. However, occlusive thrombus in the distal segment of the right coronary artery (RCA) was visualized, with distal TIMI 1 flow (Figures 1 A, B). 5000 IU of unfractionated heparin was administered, manual thrombectomy was performed (Figure $1 \mathrm{C}$ ) and the TIMI 3 flow was restored (Figure $1 \mathrm{D}$ ). After successful thrombus evacuation there was no RCA stenosis on angiography. Thus, neither balloon angioplasty nor stenting was attempted. Echocardiography revealed akinesis of the inferior wall, modestly impaired left ventricular systolic function with ejection fraction of $50 \%$ and moderate mitral regurgitation secondary to perforation of the posterior mitral leaflet (probably old), which was decided to be left for conservative treatment. Antithrombotic treatment with $75 \mathrm{mg}$ of clopidogrel and $1 \mathrm{mg} /$ kg o.d. of enoxaparin was prescribed indefinitely.

Breast cancer may itself induce a hypercoagulatory state, which can subsequently lead to thrombus forma- tion within the venous system, pulmonary circulation, and more rarely within different arteries. Antithrombotic prophylaxis with low-molecular-weight heparin (LMWH) is recommended, and its withdrawal for any reason may exacerbate the hypercoagulable tendency.

The role of thrombectomy as an adjunctive tool for primary percutaneous coronary intervention after ambivalent results of the four main randomized trials [see insightful comments of Musiałek [1] on the limitations of these studies] remains uncertain. Surprisingly, neither thrombus burden nor the coronary flow before interventional treatment had any significant impact on the outcome in the TASTE trial [2]. However, the vast majority of the patients included in these trials had underlying atherosclerosis, in contrast to our patient.

Secondly, an intact artery after successful thrombectomy does not need balloon angioplasty or stent implantation, unless relevant intraluminal abnormality is seen. As suggested by Souteyrand et al., deferred twostep treatment of ACS with optical coherence tomography (OCT) guidance may lead to stenting abstention in more than $30 \%$ of cases after large thrombus removal with thrombectomy [3]. Thrombus regression under optimal pharmacotherapy has also been confirmed by other OCT studies [4]. Stenting by itself may lead to thrombosis. Although the causal relation between malignancy and stent thrombosis is not statistically proven, several reports of such a complication have been published [5]. Furthermore, even in the era of drug-eluting stents, possible restenosis should be taken into account. Finally, stent implantation imposes a temporary regimen of double antiplatelet therapy. Combination of such pharmacotherapy with LMWH may not be the optimal option, as it increases the bleeding risk.

In conclusion, recent ESC guidelines have downgraded the thrombectomy recommendations for STEMI

\section{Corresponding author:}

Paweł Tyczyński MD, PhD, Department of Interventional Cardiology and Angiology, Institute of Cardiology, 42 Alpejska St, 04-628 Warsaw, Poland, phone: +48 696091079 , e-mail: medykpol@wp.pl

Received: 1.06.2015, accepted: 26.07.2015. 

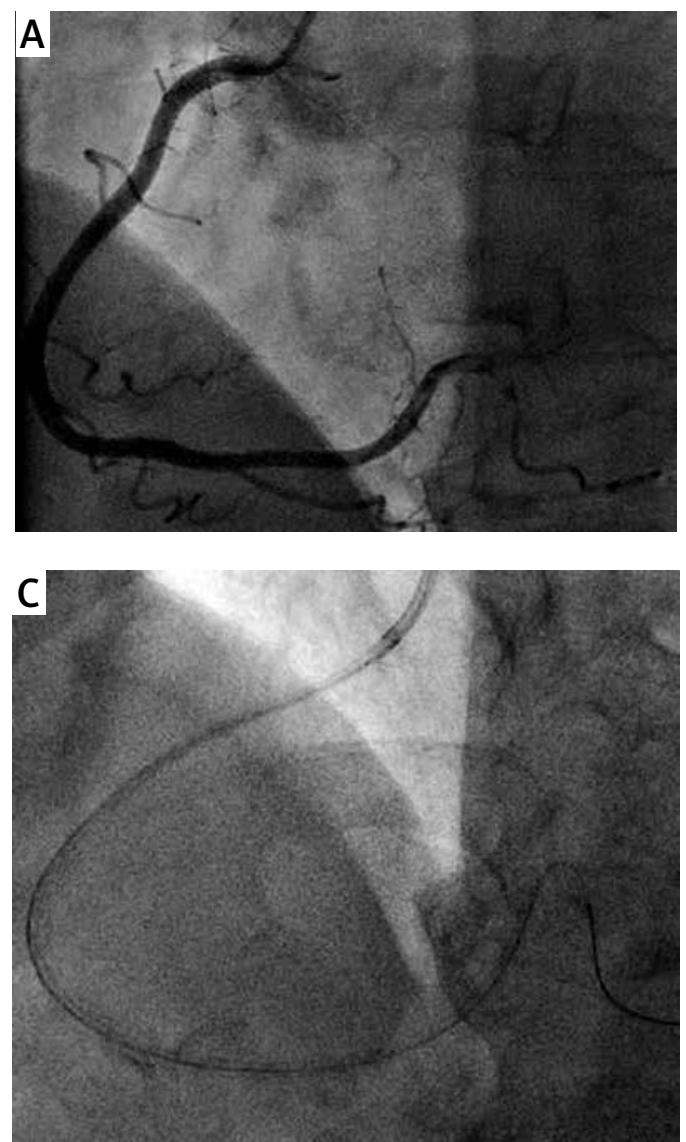
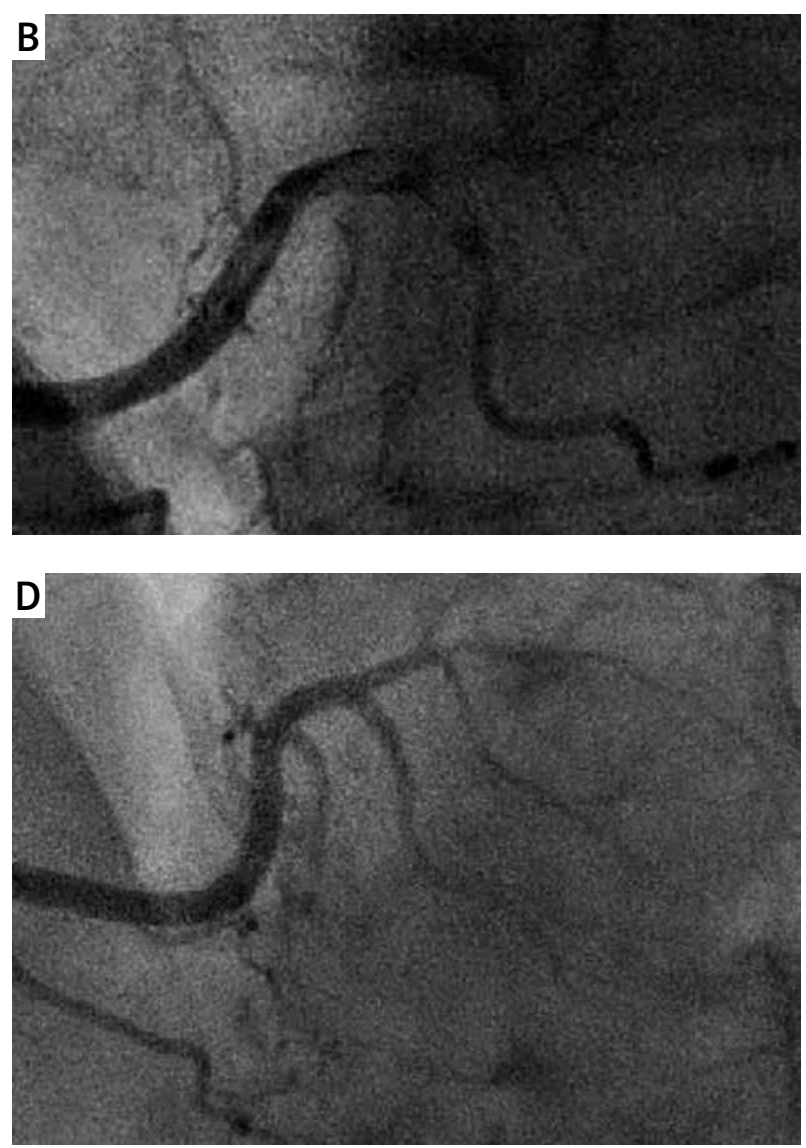

Figure 1. A - Angiography of the right coronary artery with distal filling defect (TIMI 1), strongly suggesting intracoronary thrombi. B - Magnification of the distal segment. C - Manual thrombectomy. D - Restored contrast flow (TIMI 3) without any intraluminal filling defects

patients to class IIb (or class Ila for stent thrombosis). However, in selected NSTEMI cases this therapy must not be forgotten, and not only as an adjunctive therapy, but even as a sole interventional tool.

\section{Conflict of interest}

The authors declare no conflict of interest.

\section{References}

1. Musiałek P. TASTE-less endpoint of 30-day mortality (and some other issues with TASTE) in evaluating the effectiveness of thrombus aspiration in STEMI: not the "evidence" to change the current practice of routine consideration of manual thrombus extraction. Kardiol Pol 2014; 72: 479-87.

2. Fröbert O, Lagerqvist B, Olivecrona GK, et al. Thrombus aspiration during ST-segment elevation myocardial infarction. N Engl J Med 2013; 369: 1587-97.

3. Souteyrand G, Amabile N, Combaret N, et al. Invasive management without stents in selected acute coronary syndrome patients with a large thrombus burden: a prospective study of optical coherence tomography guided treatment decisions. Eurolntervention 2015; 11: 895-904.
4. Amabile N, Hammas S, Fradi S, et al. Intra-coronary thrombus evolution during acute coronary syndrome: regression assessment by serial optical coherence tomography analyses. Eur Heart J Cardiovasc Imaging 2015; 16: 433-40.

5. Lee JM, Yoon CH. Acute coronary stent thrombosis in cancer patients: a case series report. Korean Circ J 2012; 42: 487-91. 